\title{
Corela
}

Cognition, représentation, langage

HS-24 | 2018

Multicultural Spoken English

\section{L'accent préfère-t-il le sens ? Les noms féminins en -ess en anglais britannique contemporain}

Jérémy Castanier

\section{OpenEdition}

\section{Journals}

\section{Electronic version}

URL: http://journals.openedition.org/corela/5291

DOI: 10.4000/corela.5291

ISSN: 1638-573X

\section{Publisher}

Cercle linguistique du Centre et de I'Ouest - CerLICO

\section{Electronic reference}

Jérémy Castanier, «L'accent préfère-t-il le sens ? Les noms féminins en -ess en anglais britannique contemporain », Corela [Online], HS-24 | 2018, Online since 25 June 2018, connection on 02 May 2019. URL : http://journals.openedition.org/corela/5291; DOI : 10.4000/corela.5291

This text was automatically generated on 2 May 2019.

\section{(c) (i) (3) (-)}

Corela - cognition, représentation, langage est mis à disposition selon les termes de la licence Creative Commons Attribution - Pas d'Utilisation Commerciale - Partage dans les Mêmes Conditions 4.0 International. 


\title{
L'accent préfère-t-il le sens ? Les noms féminins en -ess en anglais britannique contemporain ${ }^{\mathbf{1}}$
}

\author{
Jérémy Castanier
}

\section{Introduction}

1 Contrairement à nombre de langues du monde, qui ont notamment recours à la flexion pour marquer le genre grammatical des noms, l'anglais contemporain, de nature peu flexionnelle, est une langue globalement neutre ou non spécifique de ce point de vue. Ainsi, les signifiants teacher, doctor ou encore guard peuvent référer aussi bien à des hommes qu’à des femmes. Il existe néanmoins une petite classe de noms possédant une terminaison explicitement féminine, à savoir -ess, comme shepherdess, actress, waitress, priestess, baroness, ambassadress, etc. Nous en dénombrons $78^{2}$, consignés dans l'English Pronouncing Dictionary (EPD) et/ou dans le Longman Pronunciation Dictionary (LPD).

2 Même si une partie des noms féminins en -ess ont une fréquence d'emploi peu importante, on peut être surpris que le statut accentuel de la terminaison -ess soit rarement abordé dans les ouvrages scientifiques et les manuels de prononciation. Par comparaison, le statut contraignant de la terminaison -igible est souvent traité dans les ouvrages (voir par exemple Trevian (2003: 71-72), Duchet (1994: 19) ou encore Ginésy (2004: 115-116)) alors qu'il ne concerne en fait que cinq mots dans EPD18 et/ou LPD3 ( corrigible, dirigible, eligible, intelligible, negligible, et les versions préfixées de ceux-ci). Les rares manuels qui mentionnent la terminaison -ess se contentent souvent de la considérer comme accentuellement neutre, au même titre que -or, sans fournir de commentaires, comme on le constate par exemple dans Ginésy (2004: 98). Les lecteurs comprendront donc par exemple qu'ils sont amenés à accentuer authoress sur la première syllabe [100], l'accent restant sur la même syllabe que dans le dérivant author [10].

3 Si cette description de -ess s'applique sans problème à l'anglais américain (où aucun changement accentuel concernant cette classe lexicale n'est à signaler), force est de 
constater que l'étudiant qui ouvrira un dictionnaire de prononciation britannique contemporaine risque d'être fort dérouté. Ainsi, EPD18 comme LPD3 accentuent authoress [100] mais aussi [201]. De plus, dans le cas de l'accentuation [100], la terminaison y est transcrite soit [-es] (prononciation principale), soit [-Is] ou [-əs] (variante) $)^{3}$. Si la situation est similaire pour hostess et murderess, on constate que l'accentuation finale est même considérée comme la plus fréquente pour deaconess et shepherdess. À l'opposé, d'autres noms ne présentent pas d'accent final, tels leopardess, sculptress et governess, même si la finale inaccentuée peut tout de même se prononcer avec une voyelle pleine au moins en variante. Pourtant, des mots comme seamstress ont une voyelle strictement réduite. Il semble donc à première vue régner un certain chaos au sein de la classe des noms féminins en -ess, à tel point que, comme le souligne F. Zumstein :

Wells (1990: 253) ne peut donc constater la simple neutralité, et ajoute le commentaire laconique suivant dans l'entrée du suffixe <-ess>: "There is great interspeaker variability in the treatment of this suffix. See individual entries. » (2007: 116)

\section{Présentation du corpus dictionnairique}

4 Afin de tenter d'appréhender la globalité du phénomène, nous avons eu recours à un corpus dictionnairique couvrant d'une part le XXe siècle, à savoir : i) les 18 éditions de l' English Pronouncing Dictionary (EPD) de Daniel Jones et ses successeurs, dont la première édition fut publiée en 1917 ; ii) les 3 éditions du Longman Pronunciation Dictionary (LPD) de John C. Wells, dont la première fut publiée en 1990 ; iii) l'Oxford Dictionary of Pronunciation for Current English (ODP) de Clive Upton et al., publié en 2001 ; iv) le Concise Pronouncing Dictionary of British and American English (CPDBAE) de Jack Windsor Lewis, publié en 1972.

5 Nous avons d'autre part consulté 23 dictionnaires des XVIII et XIXe siècles, notamment le Critical Pronouncing Dictionary and Expositor of the English Language de John Walker, publié en 1791 (dont nous avons utilisé l'édition de 1842). Toutefois, la parfaite homogénéité accentuelle qui y figure rend inutile de tous les citer spécifiquement dans cette étude. Ils figurent néanmoins dans la bibliographie.

\section{Situation antérieure au XXe siècle}

Aucun des 23 dictionnaires antérieurs au XXe siècle dans lesquels nous avons systématiquement vérifié l'accentuation des 78 noms féminins en -ess étudiés (lorsqu'ils étaient présents) n'a révélé l'existence d'une accentuation finale. Il semblerait donc que le développement du schéma oxyton (accent final) de ces noms ait eu lieu au XXe siècle. On ne trouve que deux notes discordantes. La première provient du dictionnaire de Walker: si celui-ci accentue bien le nom princess sur la première syllabe, il affirme cependant dans son principe 502 :

Hence we may perceive the glaring absurdity which prevails even in the first circles; that of pronouncing the plural of princess, and even the singular, with the accent on the second syllable, like success and successes; for we might as well say, dutchéss, and dutchésses, as, princéss and princésses; nor would a correct ear be less hurt with the latter than the former. (Walker 1842, principe 502) 
7 La seconde note discordante, qui concerne également princess, provient de Worcester $(1860)^{4}$, qui ne fournit exceptionnellement pas de prononciation pour ce nom mais indique en note :

Dr. Latham says, “The feminine form of prince is accented princ-cess', while peer'ess, priest'ess, etc. carry the accent on the first syllable. Princess is remarkable as being the only word in English where the accent lies on the subordinate syllable". - Yet no English orthoepist, so far as we know, places the accent on the second syllable. (Worcester 1860, princess; accent placé après la syllabe avant le XXe siècle)

Bien qu'elle ne soit jamais indiquée dans les dictionnaires et bien que possiblement marginale, il n'est donc pas inenvisageable que l'accentuation finale de princess ait pu naître avant le XXe siècle. Il est cependant difficile de dire s'il s'agit là d'un phénomène lié à son statut de nom féminin ou bien, plus vraisemblablement, à son statut d'emprunt au français.

La prononciation de la voyelle en position inaccentuée est difficilement appréhendable dans les dictionnaires antérieurs au XXe siècle. En effet, d'une part, une partie d'entre eux fournissent seulement l'accentuation des mots et non leur prononciation. D'autre part la plupart de ceux qui fournissent leur prononciation ne possèdent pas de système permettant de représenter les voyelles faibles, en particulier le schwa, et notent l'équivalent de [e] dans toutes les positions.

10 Quelques rares dictionnaires semblent pourtant opérer une distinction entre voyelle pleine et voyelle réduite, à l'image de Worcester (1860), qui note soit $\breve{E}$ pour la voyelle qualifiée de "short», soit Ẹ pour celle qualifiée de "obscure», cette dernière étant équivalente à [ə] ou [I]. C'est toutefois une distinction mécanique qui est effectuée dans le cas des noms féminins en -ess, puisque tous ceux qui prennent l'accent sur la pénultième (tels heiress, instructress) ont une voyelle finale transcrite E tandis que tous ceux qui ont l'accent avant la pénultième (tels lioness, ambassadress) ont une voyelle finale transcrite $\breve{E}$. Cette opposition est d'ailleurs systématique au point de transcrire traitress avec E et traitoress avec $\breve{\mathrm{E}}$.

11 Buchanan (1766) distingue également les deux prononciations, en optant soit pour ĕ, soit pour $\breve{~}$. La distinction ne semble cependant pas fiable dans ce dictionnaire car $\breve{~ e s t ~ u t i l i s e ́ ~}$ pour tous les mots depuis le début du dictionnaire jusqu'à peeress, tandis que 8 mots sur 17 en fin de dictionnaire (de poetess à votaress) sont transcrits avec ĕ, ce qui laisse supposer qu'un changement dans la logique transcriptionnelle a dû être opéré en cours d'édition du dictionnaire. Aucune logique ne nous semble par ailleurs présider au choix de ě ou de $\breve{~ d a n s ~ l a ~ p a r t i e ~ f i n a l e . ~}$

Boyer (1827), dictionnaire destiné à un lectorat francophone et fournissant des transcriptions orthographiques devant être lues «à la française ", distingue également les deux prononciations, qu'il note -ece et -ice. Presque tous les noms féminins en -ess y sont cependant transcrits avec -ece, à l'exception notable de archduchess (duchess est absent du dictionnaire), mistress et schoolmistress. Quant à Walker (1842), il transcrit tous les mots avec e2 (correspondant à [e]), à l'exception également notable de heiress, mistress et seamstress, qui sont transcrits avec i2 (correspondant à [I]). Il ajoute par ailleurs la note suivante sous l'entrée lioness:

There is a propensity pretty general of pronouncing the $e$ in this and similar words like short i: but this pronunciation, however pardonable in light colloquial speaking, would be inexcusable in reading or deliberate speaking. (Walker 1842, lioness) 
13 Walker reconnaît donc explicitement l'existence des deux prononciations [e] et [I] dans ces mots. Toutefois, la pertinence de sa remarque est fortement limitée par le fait que, de façon générale, Walker et nombre de ses contemporains fournissaient des transcriptions prescriptives reflétant bien davantage la parole publique que la conversation spontanée.

\section{Situation quantitative au XXe siècle}

14 Le dépouillement de tous nos dictionnaires publiés au XXe siècle, et en particulier des 18 éditions de l'English Pronouncing Dictionary, permet de faire apparaître le caractère progressif de la diffusion de la prononciation [-es] et du statut accentué de la terminaison -ess. Cette évolution est exposée dans l'illustration 1. Le tableau présenté en illustration 2 , lui, récapitule quantitativement les différentes possibilités pas à pas.

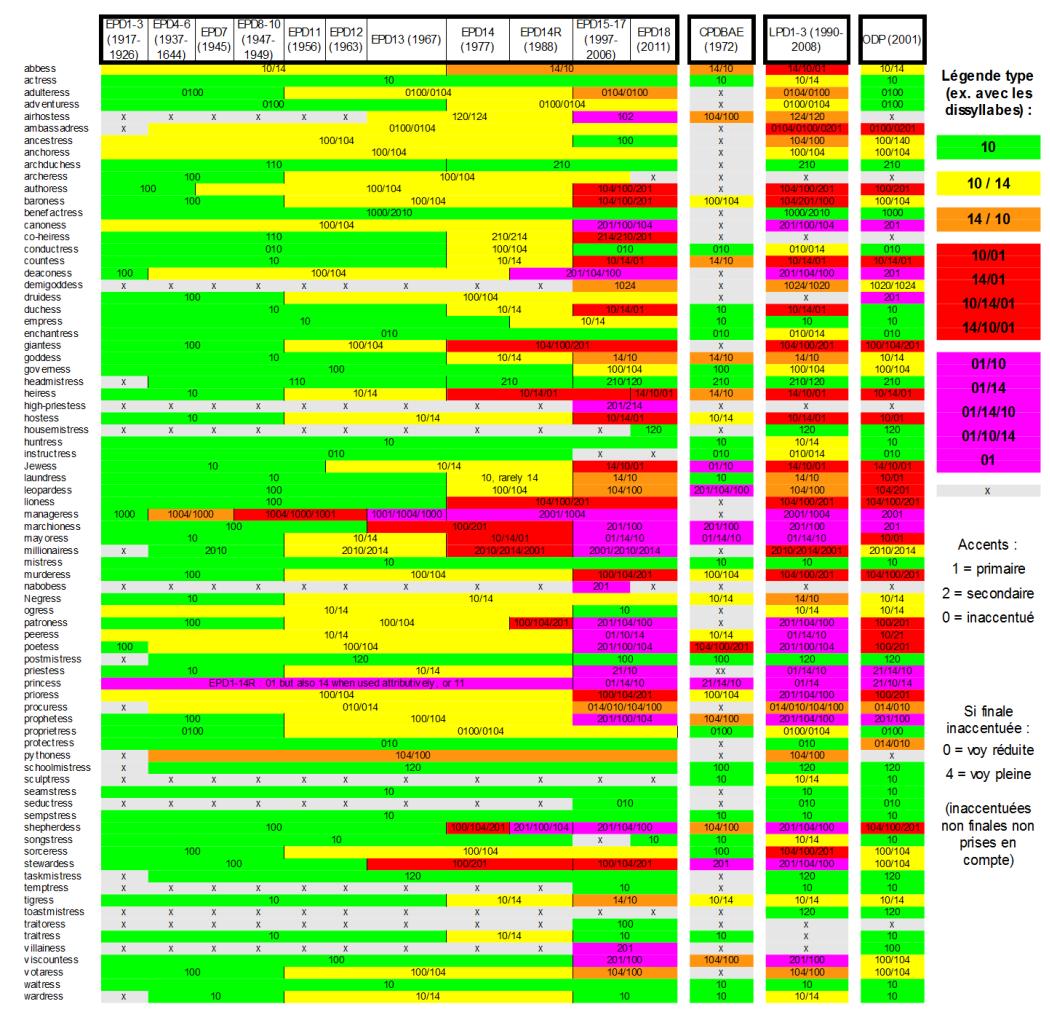

Illustration 1 : Totalité du corpus de travail accentué au XXe siècle, selon les dictionnaires. Pour l' English Pronouncing Dictionary, la série se lit diachroniquement de gauche à droite. Pour faciliter la lecture, chaque type d'accentuation est associé à une couleur unique. En cas d'impression, il est conseillé d'imprimer en couleur.

15 Pour chaque schéma accentuel, le décompte du nombre de syllabes se fonde sur une conception phonétique. La légende fournie est réutilisable quel que soit le nombre de syllabes car les couleurs codent uniquement le statut de la syllabe finale : inaccentuée réduite, inaccentuée pleine ou accentuée, et les combinaisons de ces trois statuts. Par exemple, le jaune correspond au schéma [10/14] d'un dissyllabe, au schéma [100/104] ou [010/014] d'un trisyllabe, etc. 


\begin{tabular}{|c|c|c|c|c|c|c|c|c|c|c|c|c|c|c|}
\hline & \begin{tabular}{|l|} 
EPD1-3 \\
$(1917-$ \\
$1926)$
\end{tabular} & $\begin{array}{l}\text { EPD4-6 } \\
(1937- \\
1644)\end{array}$ & $\begin{array}{c}\text { EPD7 } \\
(1945)\end{array}$ & $\begin{array}{c}\text { EPD8-10 } \\
(1947- \\
1949)\end{array}$ & $\begin{array}{l}\text { EPD11 } \\
(1956)\end{array}$ & $\begin{array}{l}\text { EPD12 } \\
\text { (1963) }\end{array}$ & $\begin{array}{l}\text { EPD13 } \\
\text { (1967) }\end{array}$ & $\begin{array}{l}\text { EPD14 } \\
(1977)\end{array}$ & $\begin{array}{l}\text { EPD14R } \\
\text { (1988) }\end{array}$ & $\begin{array}{c}\text { EPD15-17 } \\
\text { (1997- } \\
2006)\end{array}$ & $\begin{array}{l}\text { EPD18 } \\
(2011)\end{array}$ & $\begin{array}{l}\text { PDBAEE } \\
1972)\end{array}$ & \begin{tabular}{l|} 
LPD1-3 \\
$(1990-$ \\
$2008)$
\end{tabular} & $\begin{array}{c}\text { ODP } \\
(2001)\end{array}$ \\
\hline chenem & (5 & $\begin{array}{c}79,1 \% \\
(53)\end{array}$ & (52) & (52) & (34) & (33) & (31) & (19) & & $\begin{array}{c}30,1 \% \\
(22)\end{array}$ & & & & $\begin{array}{l}2,8 \% \\
29\end{array}$ \\
\hline ou $[-14]$ & $12,1 \%(7)$ & $\begin{array}{c}16.4 \% \\
(11)\end{array}$ & $\begin{array}{c}17,9 \% \\
(12)\end{array}$ & $\begin{array}{c}17,9 \% \\
(12)\end{array}$ & $\begin{array}{c}44.8 \% \\
(30)\end{array}$ & $\begin{array}{c}46,3 \% \\
(31)\end{array}$ & $\begin{array}{c}47,1 \% \\
(32)\end{array}$ & $\begin{array}{c}54,4 \% \\
(37)\end{array}$ & $\begin{array}{c}52,9 \% \\
(36)\end{array}$ & $\begin{array}{c}13,7 \% \\
(10)\end{array}$ & $12,3 \%(9)$ & $16,3 \%(7)$ & $20 \%(14)$ & $\begin{array}{c}21,1 \% \\
(15)\end{array}$ \\
\hline schen & $0 \%(0)$ & $3 \%(2)$ & $3 \%(2)$ & $1,5 \%(1)$ & $1,5 \%$ (1) & $1,5 \%$ & $1,5 \%$ & $2,9 \%(2)$ & $2,9 \%(2)$ & $\begin{array}{c}13,7 \% \\
(10)\end{array}$ & $\begin{array}{c}13,7 \% \\
(10)\end{array}$ & $3,6 \%(8)$ & $\begin{array}{c}15,7 \% \\
(11)\end{array}$ & $8 \%(2)$ \\
\hline (Total & $\begin{array}{r}98,3 \% \\
(57) \\
\end{array}$ & $\begin{array}{c}98,5 \% \\
(66) \\
\end{array}$ & $\begin{array}{c}98,5 \% \\
(66) \\
\end{array}$ & $\begin{array}{c}98,5 \% \\
(65) \\
\end{array}$ & $97 \%(65)$ & $97 \%(65)$ & $\begin{array}{c}94,1 \% \\
(64) \\
\end{array}$ & $\begin{array}{r}85,3 \% \\
(58) \\
\end{array}$ & $\begin{array}{c}82,4 \% \\
(56) \\
\end{array}$ & $\begin{array}{c}57,5 \% \\
(42) \\
\end{array}$ & $\begin{array}{r}58,9 \\
(43 \\
\end{array}$ & $\begin{array}{l}3,7 \% \\
36) \\
\end{array}$ & $\begin{array}{c}58,6 \% \\
(41)\end{array}$ & $\begin{array}{c}64,8 \% \\
(46) \\
\end{array}$ \\
\hline ante & $0 \%(0)$ & $0 \%(0)$ & $0 \%(0)$ & $1.5 \%$ (1) & $1.5 \%$ & $1.5^{\circ}$ & 2 & $11.8 \%(8)$ & 3) & $\begin{array}{c}17,8 \% \\
(13)\end{array}$ & & $2.3 \%$ (1) & $20 \%(14)$ & $\begin{array}{c}23,9 \% \\
(17)\end{array}$ \\
\hline iccer & $1,7 \%(1)$ & $1,5 \%(1)$ & $1,5 \%$ (1) & ) $1,5 \%$ (1) & $1,5 \%(1)$ & $1,5 \%(1)$ & $2,9 \%(2)$ & $2,9 \%(2)$ & $5,9 \%(4)$ & $\begin{array}{r}20, \\
(1\end{array}$ & $\begin{array}{r}20, \\
(1\end{array}$ & $11,6 \%(5)$ & $21,4 \%$ & $4,2 \%$ \\
\hline cce & $0 \%(0)$ & $0 \%(0)$ & $0 \%(0)$ & $0 \%(0)$ & $0 \%(0)$ & O\% (0) & $0 \%(0)$ & $0 \%(0)$ & $0 \%(0)$ & $4,1 \%(3)$ & $2,7 \%$ & $2,3 \%$ (1) & $0 \%(0)$ & $7 \%(5)$ \\
\hline (Total acc ent final pos sible) & $1,7 \%(1)$ & $1,5 \%(1)$ & $1,5 \%(1)$ & $3 \%(2)$ & $3 \%(2)$ & $3 \%(2)$ & $5,9 \%(4)$ & $\begin{array}{c}14,7 \% \\
(10)\end{array}$ & $\begin{array}{c}17,6 \% \\
(12)\end{array}$ & $\begin{array}{c}42,5 \% \\
(31)\end{array}$ & $\begin{array}{r}41,1 \% \\
(30)\end{array}$ & $\mid 16,3 \%(7)$ & $\begin{array}{c}41,4 \% \\
(29) \\
\end{array}$ & $\begin{array}{c}35,2 \% \\
(25)\end{array}$ \\
\hline tert & & 67 & & & & & 68 & 68 & 68 & & & & & 71 \\
\hline
\end{tabular}

Illustration 2 : Répartition des différentes réalisations phonétiques de la terminaison féminine -ess (statut accentué ou inaccentué, voyelle pleine ou réduite si inaccentuée) dans les dictionnaires du XXe siècle.

Il apparait très clairement qu'à mesure que l'on progresse dans les premières éditions de l'EPD, la terminaison est globalement inaccentuée mais passe peu à peu d'une voyelle réduite à une voyelle pleine. Seul princess peut prendre l'accent sur la finale dans EPD1-3. Alors que seuls deux à trois mots peuvent optionnellement prendre l'accent sur la finale jusqu'à EPD12, quelques mots supplémentaires peuvent ensuite le prendre dans EPD13, mais surtout dans EPD14 et EPD14R ${ }^{5}$. Enfin, ce nombre explose soudainement à partir de EPD15, jusqu'à représenter environ $41 \%$ du total, que cela soit dans EPD18 ou dans LPD3 ${ }^{6}$.

L'étendue du phénomène pousse ainsi certains linguistes à établir un parallèle entre -ess et certaines terminaisons contraignantes attirant l'accent sur elles-mêmes. C'est ce que fait Trevian, qui s'en tient toutefois au stade de la description et se contente de comparer -ess avec les terminaisons françaises (en référence à l'accentuation finale démarcative du français) :

Si la plupart ont un accent conforme à leur dérivant [...], -ess rejoint quasisystématiquement en variante le statut de -ee et -ese en admettant une accentuation du suffixe. [...] Le suffixe -ess constitue typiquement une zone de conflit de la morphophonologie anglaise, opposant isomorphisme et accentuation « à la française ». Au vu des données livrées par LPD et/ou EPD15, une conversion de [ces] items [...] à la seconde tendance semble se dessiner en anglais contemporain. (2003: 30-31; nous soulignons)

J.-M. Fournier, lui, semble établir un lien de causalité plus explicite avec les terminaisons françaises à consonne double du type finesse, cigarette, etc. :

Une mention spéciale doit être faite à propos du suffixe féminin -ess : normalement neutre, il admet néanmoins très souvent une variante en /-1/ sous l'influence de la terminaison contraignante $-C^{\prime} C^{\prime} \mathbf{e}[. .$.$] . Une prononciation sans déplacement$ d'accent par rapport au dérivant est toutefois toujours attestée. $(2010: 38$; nous soulignons)

19 Malgré tout, il est difficile de considérer le phénomène homogène, puisqu'il ne concerne qu'environ $41 \%$ des items à l'étude. Non seulement $59 \%$ des mots ne prennent pas l'accent sur la finale, mais ce sont même 23 à 33 \% qui voient leur terminaison prononcée avec une voyelle strictement réduite. L'hypothèse d'un lien avec une étymologie française est par ailleurs peu vraisemblable si l'on considère l'origine des noms féminins auxquels nous nous intéressons. En effet, certains peuvent s'accentuer [-1] et sont bien d'origine française (princess, lioness, marchioness, etc.) tandis que d'autres, qui ne sont pas d'origine française, n'ont pas d'accent final ('seamstress, 'goddess, etc). Mais on trouve aussi des 
emprunts au français ne présentant pas d'accent final (en'chantress, 'tigress, etc.), tout comme des noms n'ayant pas été empruntés au français mais pouvant prendre l'accent final (manageress, stewardess, etc.).

Quelques paires permettent d'apporter des éléments de raisonnement. D'un côté, la paire duchess [10/01] vs duchesse [01], où duchesse est emprunté au français mais n'est pas un mot à sens féminin (il désigne un meuble), montre que seule -esse peut véritablement être qualifiée de terminaison française attirant l'accent, même si l'on pourrait envisager une influence de duchesse sur duchess. D'autre part, les paires marquis [10] vs marquess [10] et fort vs fortress [10] montrent que la terminaison -ess n'attire pas l'accent, au moins dans ces cas-là, si elle n'est pas féminine (marquess désigne un marquis et non une marquise).

\section{L'importance de la morphologie}

21 L'examen minutieux du corpus lexical montre que la présence ou l'absence d'accent final est en fait très largement conditionnée par le statut morphologique de la terminaison -ess . Ainsi, deux grands cas de figure doivent être distingués :

22 - Soit la terminaison -ess est strictement suffixale et conduit à l'ajout d'une syllabe supplémentaire (dérivés imparisyllabiques) sans modification du dérivant (lion > lioness, author > authoress, poet > poetess, millionaire > millionairess, etc.) ;

23 - Soit la terminaison -ess, qu'elle soit strictement ou non un suffixe ajouté à une base existante, ne conduit pas à l'ajout d'une syllabe supplémentaire (dérivés parisyllabiques). On peut distinguer trois sous-cas de figure : 1 ) il y a substitution suffixale transparente ( governor > governess, procuror > procuress, etc.) ou plus opaque (anchorite > anchoress, abbot > abbess, etc.) ; 2) il y a syncope syllabique (hunter > huntress, warder > wardress, actor > actress, etc.) ; 3) la forme féminine n'est pas strictement dérivée de la forme masculine (duke $>$ duchess, seducer $>$ seductress ${ }^{7}$ ).

24 Le classement du corpus lexical en fonction de ces deux grands cas de figure permet alors de faire apparaître deux comportements accentuels radicalement différents, puisque seul le premier grand cas de figure (ajout transparent d'une syllabe) est fortement sujet à l'accentuation finale, le second y étant très réfractaire. La situation est présentée dans l'illustration 3, où la partie supérieure correspond aux cas d'ajout transparents tandis que la partie inférieure correspond aux cas où dérivants et dérivés sont parisyllabiques. 


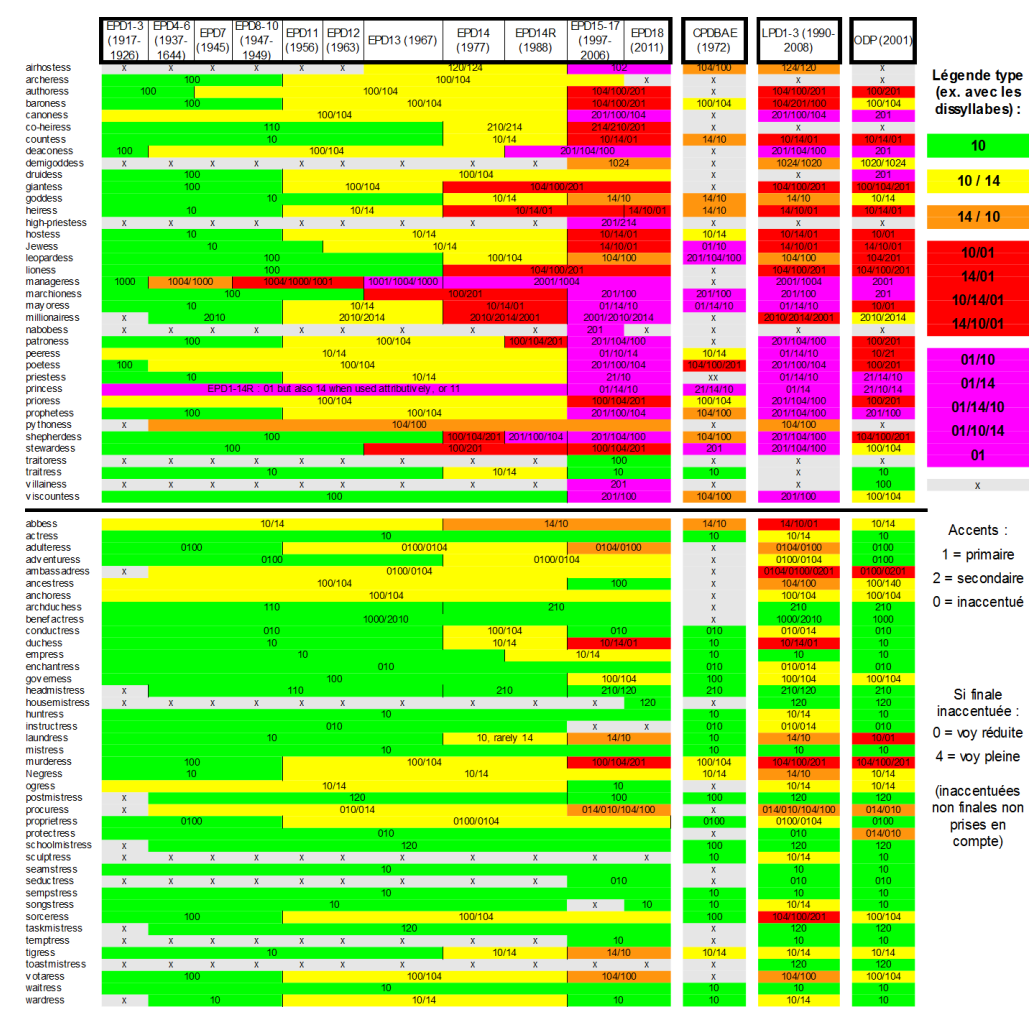

Illustration 3 : Corpus de travail au XXe siècle trié par type morphologique : dérivés parisyllabiques dans la partie inférieure, et dérivés où -ess représente une syllabe supplémentaire dans la partie supérieure.

Le fait qu'il s'agisse ou non d'un ajout transparent semble globalement suffire à expliquer la présence ou l'absence de l'accent final. Ainsi, en cas d'ajout transparent, l'accentuation finale n'entrave en rien l'isomorphisme vocalique entre dérivants et dérivés (cf. Dahak 2011 : 206), comme dans [kaont] > [,kaon'tess]. Elle n'entrave pas non plus l'application de la règle dérivationnelle dans les dérivés, à savoir la transformation de l'accent primaire du dérivant en accent secondaire du dérivé : lion [10] > lioness [201], shepherd [10] $>$ shepherdess [201], etc. La règle s'applique aussi à millionaire [201] > millionairess [2001] (où la voyelle pénultième conserve d'ailleurs sa valeur pleine), sur le même modèle que represent [201] > representation [20010]. Dans le cas des dérivés de monosyllabes, tels que host [1] > hostess [21], le phénomène est le même qu'avec les dérivés classiques du type trust [1] > trustee [21].

En revanche, dans le cas des dérivants en [010], la syncope empêche l'accentuation finale car elle reviendrait à contredire la règle dérivationnelle, et donc à empêcher l'isomorphisme vocalique entre dérivants et dérivés: conductor, instructor [010] > conductress, instructress [010] et non [201] comme dans adore [01] > adoration [2010]. On voit que l'ajout d'une syllabe supplémentaire constitue ici une condition nécessaire à l'accentuation finale, alors que le rare schéma [021] est pourtant régulièrement attesté par ailleurs avec des dérivations telles que escape [01] > escapee [021] ou [201] (on aurait donc pu s'attendre à instructress [021]). Les dérivations du type conductor > conductress se comportent donc en fait de la même façon qu'avec l'accentuation principale [010] de employee, dérivé de employ [01]. Plus largement, l'isomorphisme accentuel s'impose entre dérivants et dérivés parisyllabiques car il permet la conservation du même schéma accentuel dans les deux cas : tiger [10] > tigress [10], adventurer [0100] > adventuress [0100], 
etc. De ce point de vue, la forme féminine n'est pas dérivée de la forme masculine mais lui est subordonnée.

relevant dans certains cas de la « pulsion ludique » évoquée par Tournier :

Il existe ainsi des mots - que l'on peut considérer comme inutiles - que l'on crée pour s'amuser, et dont le caractère insolite, cocasse, pittoresque, fantaisiste, leur permet de conquérir droit de cité dans la langue de la communauté ou, au moins, d'une fraction de la communauté. Le langage humain est aussi un terrain de jeu et l'homme éprouve le besoin de jouer sur ce terrain comme sur d'autres : le troisième ressort de la création lexicale est la pulsion ludique. (2004:196) (2004: 198). Il nous semble en fait possible de situer -ess comme appartenant au même ensemble lexicogénique que le trio -er/-ee/-or, comme dans certaines paires, lexicalisées ou non, souvent utilisées pour illustrer les rôles sémantiques en syntaxe : bailor / bailee, giver / givee, etc. On peut ainsi créer à souhait des singeress, paintress ou encore philosopheress. 

partie des noms féminins en -ess. Cela est souligné par Hellinger:

Frequently, the creation of female-specific nouns will produce semantically asymetrical pairs in which the female represents the lesser category, illustrating what Schulz (1975) has called semantic derogation. (2006: 267)

Holmes illustre précisément le caractère péjoratif de la dérivation:

One can [...] regard -ess as signalling women's secondary status - as marking a deviation from the (male) norm. Baron (1986), for example, comments that "in most instances, the use of the feminine suffix implies that the feminine noun derives from the masculine and that it is of secondary or inferior status". [...] Few would deny that someone introduced as a philosopheress is unlikely to be treated with respect. [...] It is impossible to treat a poetess as a serious writer, and no one would think of labeling Janet Frame an authoress or Kiri Te Kanawa a songstress. (1993 : 360)

Dans le domaine littéraire précisément, les noms authoress et poetess ont attiré beaucoup d'attention, notamment parce qu'il appartenait justement aux femmes poètes et écrivains d'utiliser ou non le terme pour se définir. Dans la seconde édition de Fowler's Modern English Usage, Gowers expliquait ainsi :

Authoress is a word that has always been disliked by authoresses themselves, perhaps on the ground that sex is irrelevant to art and that the word implies disparagement of women's literary abilities. [...] When the OED was published the authoresses seemed to be getting their way; for the opinion is there given that authoress "is now only used when sex is purposely emphasized; otherwise in all the senses, and especially the last [sc. a female literary composer], author is now used of both sexes". (1965, entrée authoress)

Dans la première édition, Fowler lui-même s'élevait encore plus fortement contre l'usage de ce type de noms :

This article is intended as a counter-protest. The authoress, poetess \& paintress, \& sometimes the patroness \& the inspectress, take exception to the indication of sex in these designations. They regard the distinction as derogatory to them \& as implying inequality between the sexes; an author is an author, that is all that concerns any reader, \& it is impertinent curiosity to want to know whether the author is male or female. (1926, entrée feminine designations)

Inversement, Elsa Greene publia en 1972 un article consacré à Emily Dickinson en tant que femme poète, qu'elle intitula "Emily Dickinson was a poetess", preuve de la controverse sur ce terme. On y lit notamment:

Emily Dickinson's exceptionally courageous poetic achievement might have taught later generations that there is no conflict between femaleness and seriousness in poetry. But most of us continue to use the term "poetess" to designate women who write "feminine poems" - meaning insipid, conventional verses; and we promote exceptional women such as Emily Dickinson to the rank of "poet". Our terminology still implies the nineteenth-century idea that femaleness limits poetic capacity. Our practice provides only that some women may be able to transcend merely female experience and thereby engage an audience in matters of universal interest. As long as we feel the need to separate a woman from her sex in order to appreciate her poetry, we perpetuate analytic categories which mutilate female subjects in the name of understanding them. For the sake of integrity, hers and our own, it is essential to remember that Emily Dickinson was a poetess. (1972:63).

Et pourtant, il existe aussi un certain nombre de noms féminins en -ess qui ne véhiculent aucune connotation négative, comme actress, heiress, shepherdess, princess, etc. Certains auteurs justifient l'existence de ces noms par l'impossibilité d'utiliser les formes sans -ess pour renvoyer à une femme, par exemple ici avec Holmes : 
In some cases it is difficult to see how the -ess form could be avoided. For a number of forms in this category, unlike most forms in the occupational category, the base form is marked as male and cannot act as a generic. Consequently, the forms baroness, duchess, empress, and princess are the only available means of expressing the meaning 'a female of the rank specified', though in fact they more often refer to 'the wife of a male of the rank specified'. It it possible that this may change, but at present the forms baron, duke, emperor, and prince are marked with the semantic feature 'male' and they cannot be used to refer to females. (1993:363)

Dans la 2e édition de Fowler's Modern English Usage, Gowers défend l'usage de certains noms pour des raisons plus larges :

It is natural that words like abbess, actress, deaconess, stewardess, wardress, should do so, for in those occupations women have duties peculiar to their sex [...]. (Gower 1965, entrée feminine designations)

\section{Interaction entre morphologie et sémantique}

Qu'il soit péjoratif ou non, il nous semble que le possible effet contrastif obtenu lors de l'ajout du suffixe -ess corresponde à une sur-spécification agentive au niveau notionnel. Par exemple, le nom baron peut se décomposer notionnellement en " titre + agent masculin ». De même, le nom shepherd se décompose en "activité + agent masculin ». Dans les deux cas, la dérivation en -ess, qui n'ampute aucun élément du dérivant, revient donc à ajouter une seconde spécification agentive, féminine cette fois. Ainsi, on peut alors décomposer baroness en " titre + agent masculin + agent féminin ». Il en va de même des dérivés de noms neutres. Par exemple, author et manager peuvent se décomposer en "activité + agent neutre ». Par conséquent, les dérivés transparents authoress et manageress se décomposent en " activité + agent neutre + agent féminin ", d'où, là aussi, une sur-spécification agentive.

Dans les deux cas, la dérivation donne donc lieu à des paires asymétriques où la forme féminine est une forme secondaire, dérivée soit du masculin, soit du neutre. Cette surspécification permet alors l'existence d'un accent final de type contrastif. Par effet de retour, il est d'ailleurs possible de réinterpréter les formes neutres comme masculines : si l'on désigne une femme auteur par le terme authoress, author en vient par effet de retour à désigner un homme.

Par opposition, la syncope et la substitution de suffixes permettent de gommer cette surspécification. Par exemple, governor se décompose en "activité + agent neutre ", or la substitution suffixale governor > governess revient à ne conserver que le dérivant verbal govern, qui ne désigne que l'activité. Par conséquent, governess se décompose seulement en « activité + agent féminin ». De la même manière, lors de la dérivation waiter > waitress, l'effacement du segment -e- conduit à effacer par la même occasion la spécification " agent masculin », le <r> étant resyllabifié en attaque branchante de la seconde syllabe. Par conséquent, waiteress se décompose en " activité + agent masculin + agent féminin ", évitant ici aussi la sur-spécification agentive.

Cette fois-ci, il y a donc création de paires symétriques, où la forme féminine n'est pas perçue comme sémantiquement dérivée du masculin ou du neutre. L'absence de surspécification agentive, par conséquent, empêche l'existence d'un accent final de type contrastif, précisément parce qu'il n'y a ici aucun contraste sur le plan de la morphologie de surface du nom féminin. 

conspirent. Ainsi, dans authoress, le suffixe -ess constitue une syllabe supplémentaire par rapport au dérivant, et construit en même temps une sur-spécification agentive. Inversement, dans waitress, il n'y a ni syllabe supplémentaire, ni sur-spécification agentive.

'exemple de governor nous semble très éclairant sur ce point. Comme le précise Holmes (1993 : 360), qui a travaillé sur le Corpus of written New Zealand English, governess n'apparaît que pour faire référence à l'éducation des enfants. Outre l'absence de sur-spécification agentive due au parisyllabisme (governess = "activité + agent féminin »), le fait qu'il s'agisse dans la réalité extra-linguistique d'un emploi occupé essentiellement par des femmes conduit la propriété « féminin » à faire partie de la notion /governess/. L'absence d'accent final contrastif en est donc d'autant plus logique. À l'inverse, on pourrait vouloir dériver governor avec le suffixe -ess dans un sens politique, pour renvoyer non pas à une gouvernante mais à une femme gouverneur (d'un État des États-Unis, par exemple). Cette fois, la propriété « féminin » ne fait probablement pas partie de la notion, ce poste étant très majoritairement occupé par des hommes. Et en effet, même si le terme n'est pas lexicalisé, on trouve via le moteur de recherche Google plus d'un millier d'occurrences du terme governoress au sens politique. La propriété «féminin" étant cette fois plus inhabituelle pour un tel poste, certains locuteurs ont donc jugé utile d'ajouter et non de substituer le suffixe -ess. Il apparaît alors probable que la prononciation d'une telle forme admette au moins une voyelle finale systématiquement pleine, si ce n'est même accentuée.

\section{Quand la sémantique prend le pas sur la morphologie}

Si nous avons vu supra que la présence ou l'absence de sur-spécification agentive se superpose normalement à la présence ou l'absence d'une syllabe supplémentaire dans la forme féminine, il existe cependant des cas d'accentuation finale dans des dérivés pourtant parisyllabiques, comme avec ambassador [0100] > ambassadress [0100] ou [0201]. On peut supposer ici que la sémantique prend le pas sur la morphologie. Ainsi, ambassadress a deux sens, désignant soit la femme d'un ambassadeur, soit une femme ambassadeur. Il parait raisonnable de penser que l'accentuation [0201] soit en fait réservée à ce dernier sens, pour lequel la propriété « féminin » est perçue comme moins évidente, puisque la fonction prime habituellement sur le sexe de la personne.

De la même façon, si governess ne possède pas le schéma [201], en revanche murderess le possède bien en variante, alors qu'il est pourtant également formé par substitution suffixale. Cependant, la propriété «féminin » est perçue comme appartenant à la notion pour governess car la fonction est majoritairement occupée par des femmes, tandis que le " genre social » (cf. Hellinger 2006: 266-267) de la personne qui commet un meurtre est habituellement masculin. Le fait que le meurtrier puisse s'avérer être une meurtrière est alors perçu avec davantage de surprise, justifiant un accent final contrastif. À l'inverse, pythoness n'admet pas d'accent final bien qu'il soit étymologiquement dérivé de python car l'Oracle de Delphe est nécessairement une femme : il n'existe pas de python occupant cette fonction. 
53 De façon plus générale, l'examen détaillé du sémantisme de tous les noms féminins en -ess permet de mettre au jour une gradation sociale corrélée au statut accentuel des noms concernés. Les différents ensembles s'inscrivant dans cette gradation sont les suivants :

54 - lexique religieux, politique et titres de noblesse (priestess, baroness, peeress, etc.) : ajout transparent de -ess et accent final majoritaire ;

55 - rôles sociaux connotés positivement (heiress, hostess, millionairess, etc.): ajout transparent de -ess mais accent final en variante seulement ;

56 - simples métiers: syncope ou substitution suffixale (actress, governess, laundress, etc.) : accentuation du radical très majoritaire; six mots font exception, dont manageress, authoress et poetess [-1], mais on a ici ajout transparent de -ess ;

57 - comportements connotés négativement (adulteress, temptress, traitress, etc.) : syncope et accent sur le radical.

58 Dans l'ensemble, on constate encore une fois que la morphologie interagit avec le sémantisme des noms concernés, mais la gradation sémantique proposée permet d'affiner la répartition de l'accent final en prononciation principale, en variante, ou bien son absence. Preuves de la prégnance du critère sémantique, les noms abbess et duchess, qui sont pourtant morphologiquement plus éloignés des formes masculines abbot (et non ${ }^{*} a b b,{ }^{*}$ abber ou *abbor) et duke (et non *duch, *ducher ou *duchor), peuvent prendre l'accent sur la finale puisqu'ils appartiennent à l'ensemble « lexique religieux, politique et titres de noblesse ». Le conflit entre morphologie et sémantique qui les concerne ne leur octroie toutefois l'accent final qu'en variante.

59 Les noms féminins désignant des animaux femelles s'inscrivent en dehors de la gradation sémantique que nous proposons car il n'est pas question ici de s'intéresser au niveau social des signifiés, ni d'y rechercher une connotation positive ou négative. Au contraire, il est naturel de distinguer le mâle de la femelle dans le règne animal, d'où l'usage de deux mots distincts sans que celui du nom féminin implique une quelconque surprise. Ainsi, si tigress s'accentue uniquement sur le radical (mais il est vrai qu'il s'agit d'un cas de syncope), il en va de même pour leopardess (un cas d'ajout transparent de -ess) et l'accent final n'affecte lioness qu'en variante ${ }^{10}$.

60 Concernant, enfin, les noms dont la voyelle finale est obligatoirement inaccentuée, on peut esquisser quelques pistes de réflexion:

61 - le trio actress, mistress et waitress s'accentue [10] et n'admet qu'une voyelle finale strictement réduite. On note par ailleurs que mistress était également transcrit avec une voyelle réduite dans Walker (1842) et Boyer (1829) (cf. supra). Il s'agit ici de noms féminins en -ess parmi les plus courants et habituellement dépourvus de toute connotation péjorative. De plus, les noms actor, master et waiter sont davantage masculins que neutres.

62 - goddess s'accentue [10], n'admet une voyelle finale pleine qu'en variante et n'admet pas d'accent final. Il s'agit toutefois du nom dont la fréquence d'emploi est la plus élevée dans toute la classe. De plus, la distinction entre dieux et déesses dans la mythologie semble naturelle.

63 - l'usage de la voyelle inaccentuée mais pleine pourrait tout de même avoir pour fonction de signaler le caractère socialement surprenant du référent. Par exemple, abbess s'accentue essentiellement [10] mais se prononce principalement avec une voyelle pleine. Une locutrice native que nous avons interrogée pour cette étude a d'ailleurs prononcé abbess avec une voyelle pleine, en justifiant explicitement sa prononciation ['æbes] plutôt 
que ['æbis] ou ['æbəs] par le fait que le milieu religieux est "male-dominated». Inversement, seamstress désigne un métier essentiellement féminin (d'ailleurs, Dahak (2011 : 244) précise qu'il n'y a pas de masculin pour sempstress et seamstress) et ne se prononce par conséquent qu'avec une voyelle réduite. Notre démonstration est néanmoins mise à mal par laundress, prononcé principalement ['lo:ndres].

\section{Un cas de diffusion lexicale?}

La progression de l'accent n'ayant pas affecté tous les items simultanément sur le plan diachronique, nous avons voulu vérifier si la classe des noms féminins en -ess pouvait avoir été sujette à un phénomène de diffusion lexicale. Ce phénomène suppose que, dans une classe de mots donnés, un petit nombre d'items sont affectés en premier, permettant ensuite que le reste de la classe (ou du moins une plus grande partie) soit à son tour affecté à un moment ultérieur.

William Yang, souvent considéré comme le promoteur du principe de la diffusion lexicale, décrit ce phénomène comme suit :

Ideally, before the change, all speakers will use sound $\mathrm{X}$ in all relevant morphemes; after the change, all speakers will use sound $Y$ in the same set of morphemes. The dimension of time may be studied in each of three relatively independent parameters : (1) phonetic, i.e. from sound X to sound Y; (2) lexical, i.e. from morpheme to morpheme in the relevant part of an individual's vocabulary ; and (3) social, i.e. from speaker to speaker in the same dialect. (1969: 12-13)

According to this view, during the early phase of the change only a small sector of the relevant morphemes is affected. Some of the affected morphemes may change to the Y-pronunciation. Other morphemes, however, will at first have both the Xpronunciation and the Y-pronunciation, fluctuating either randomly or according to some such factor as tempo or style. [...] But the X-pronunciation will gradually be suppressed in favor of the Y-pronunciation. These doublets, then, serve as a kind of psychological bridge between the two end-points of a sound change, carrying along with them even those morphemes which do not go through a doublet stage. (1969: 15)

On voit en effet que le changement qui nous concerne est ici progressif sur le plan diachronique, puisque tous les items s'accentuaient sur le dérivant au départ (même si princess nous empêche de dater très précisément ce « départ »), et qu'ils sont de plus en plus nombreux à posséder à présent plusieurs prononciations. Preuve cependant que le processus est encore en cours, l'accentuation finale n'est la seule accentuation possible que pour trois mots selon EPD17 (cinq mots selon ODP, aucun selon LPD). Yang précise en effet:

Since living languages are constantly undergoing change, we should expect to find many seeming exceptions to changes which have not completed their course. These forms are not true residues, in one sense, even though we cannot specify them in general terms either phonetically or morphologically, since in time the appropriate phonological changes will reach them and make them regular. (1969: 10)

On voit ainsi que, pour le moment, la plupart des noms féminins en -ess où -ess représente un ajout transparent possèdent encore une accentuation isomorphe par rapport au radical. Surtout, la plupart des cas où -ess ne représente pas un ajout transparent ne sont pas affectés par l'accent final. Il n'est donc pour le moment pas encore sûr qu'il faille en fait considérer que le phénomène s'applique aux noms féminins dérivés par -ess en général, ou bien seulement à la sous-classe des dérivés transparents. Le fait que certains 
cas de substitution ou de syncope commencent à être affectés pour des raisons sémantiques permet toutefois de suspecter que le changement pourrait se poursuivre dans l'avenir au sein de la classe toute entière.

D'autre part, une question récurrente dans la description du changement linguistique est de savoir si ce changement est abrupt ou graduel. Yang explique ainsi:

The process of diffusion within a speaker's vocabulary may likewise be thought of as being either abrupt (i.e., all relevant morphemes change 'simultaneously') or gradual (i.e., the change affects the relevant morphemes severally in succession.) There are then four logical possibilities in viewing how a sound change operates on an individual's vocabulary :

(1) phonetically abrupt and lexically abrupt ; (2) phonetically abrupt and lexically gradual ; (3) phonetically gradual and lexically abrupt ; (4) phonetically gradual and lexically gradual

Of the two possibilities which involve a phonetically abrupt implementation, (1) is refuted by elementary observations [...]. (3) is the view taken by the neogrammarians and continues to be widely accepted today. The hypothesis of lexical diffusion stipulates (2) and (4). Of these two, (2) is the more compelling : for given that the phonetic implementation is abrupt, and that an individual's vocabulary does not change all that suddenly, the obvious conclusion is that what actually takes place is a kind of diffusion from morpheme to morpheme in this vocabulary. This diffusion within a lexicon is basically the same mechanism as the more observed forms of diffusion across dialects or languages, and diffuses only in its scope or operation; lexical diffusion is more local, the other forms are more global. (1969: 14-15).

69 Sur le plan phonétique, Yang défend le caractère abrupt du changement :

There is good reason to believe that many types of sound change must be regarded as operating at a phonological level that is much more abstract than the phonetic level, and therefore phonetically non-gradual. So, for a word like 'acclimate' in which the pronunciation changes from [əklájmit], the only pronunciation found in some older dictionaries, to [áklimejt], where all three vowels are different (in addition to the difference in accent pattern), it is surely unrealistic to suppose that there was a gradual and proportionate shift along all four phonetic dimensions.

(1969: 14)

70 Si nous adhérons de façon générale à ce point de vue en ce qui concerne le changement accentuel, le changement qui nous occupe ici semble en revanche bel et bien graduel. En effet, nous avons vu que, dans une grande partie des cas, le passage d'une voyelle finale réduite à une voyelle finale accentuée se fait via un stade intermédiaire où la voyelle est inaccentuée mais pleine :['dzaIəntIs] > ['ḋaiəntes] > [,dzaIən'tes]. Ce caractère graduel est justement permis par l'isomorsphisme vocalique (dans le reste du mot) mentionné supra. D'autre part, le passage de ['ḋaiəntes] à [,dzaiən'tes] n'est pas nécessairement brutal, puisqu'il ne s'agit ici que d'une modification du degré d'accentuation de la première et de la troisième syllabes, étant entendu que tous les degrés accentuels ne peuvent être rendus par la simple notation [0], [2] et [1]. On pourrait par exemple utiliser le système de notation par chiffres pour symboliser le poids relatif en termes acoustiques (intensité, fréquence fondamentale, etc.) entre deux syllabes accentuées. Dans le cas illustratif de giantess, on pourrait ainsi très bien noter [102], [103], [104], etc. selon le degré d'accentuation de la syllabe finale, mais aussi [305], [201], [302], [704], etc. selon la différence relative d'accentuation entre les deux syllabes. On peut tout aussi bien s'attendre à une accentuation égale du type [101 ${ }^{11}$. 
71 Un point cependant qui n'est pas traité par Yang dans son article de 1969, et pour lequel il préfère renvoyer à d'autres auteurs, est celui de la cause initiale de l'apparition du changement ("without concerning ourselves with the intriguing question of how the change came into the language in the first place » $1969: 12)$. Nous considérons pour notre part que la cause de l'apparition du changement fait partie intégrante du phénomène de diffusion lexicale et qu'il est donc essentiel de tenter de la reconstituer. Nous avons par ailleurs eu l'occasion d'identifier plusieurs phénomènes de cette nature dans Castanier (2010) et Castanier (2016) en isolant précisément certains mots précurseurs, et nous avons pu reconstituer certaines causes. Par exemple, une fréquence d'emploi élevée explique l'initiation d'un processus de rétraction accentuelle au sein de la classe des mots en -ette, visiblement introduit par le biais de etiquette [201] > [100] (cf. Castanier 2016 : 202-205). Il en va de même pour la progression de l'accent vers la syllabe antépénultième des adverbes en -arily [-100] en anglais britannique (cf. Castanier $2016: 560-572^{12}$ ), mais ce critère fréquentiel s'est ici surtout doublé d'un critère sémantique. En effet, les mots initiateurs $\mathrm{du}$ changement dans cette classe (necessarily, puis primarily, puis enfin diffusion à la quasi-totalité du reste de la classe) sont ceux qui admettent un sens figuré ${ }^{13}$ susceptible de recevoir une accentuation affectée ou emphatique.

72 Ainsi, parmi les cas où le suffixe -ess est un ajout transparent, on constate sur le plan diachronique que le premier mot ayant acquis l'accentuation finale (en dehors du cas délicat de princess, qui est aussi d'origine française) est manageress, dans EPD8 (1947), qui avait même une voyelle pleine depuis EPD4 (1937). Il fut notamment suivi par stewardess, dans EPD13 (1967). Ces cas sont mis en évidence dans l'illustration 4. Manageress fut également le premier à adopter l'accent final en prononciation principale, à savoir dans EPD13 (1967). A contrario, la plupart des mots qui ont acquis un accent final l'on fait à partir de EPD14 (1977) et surtout de EPD15 (1997). Dans tous ces cas, il faut bien entendu tenir compte du délai nécessaire entre l'apparition des formes dans la langue et leur consignation lexicographique, ce qui permet de supposer que manageress a dû commencer à être affecté par ce phénomène au tout début du XXe siècle.

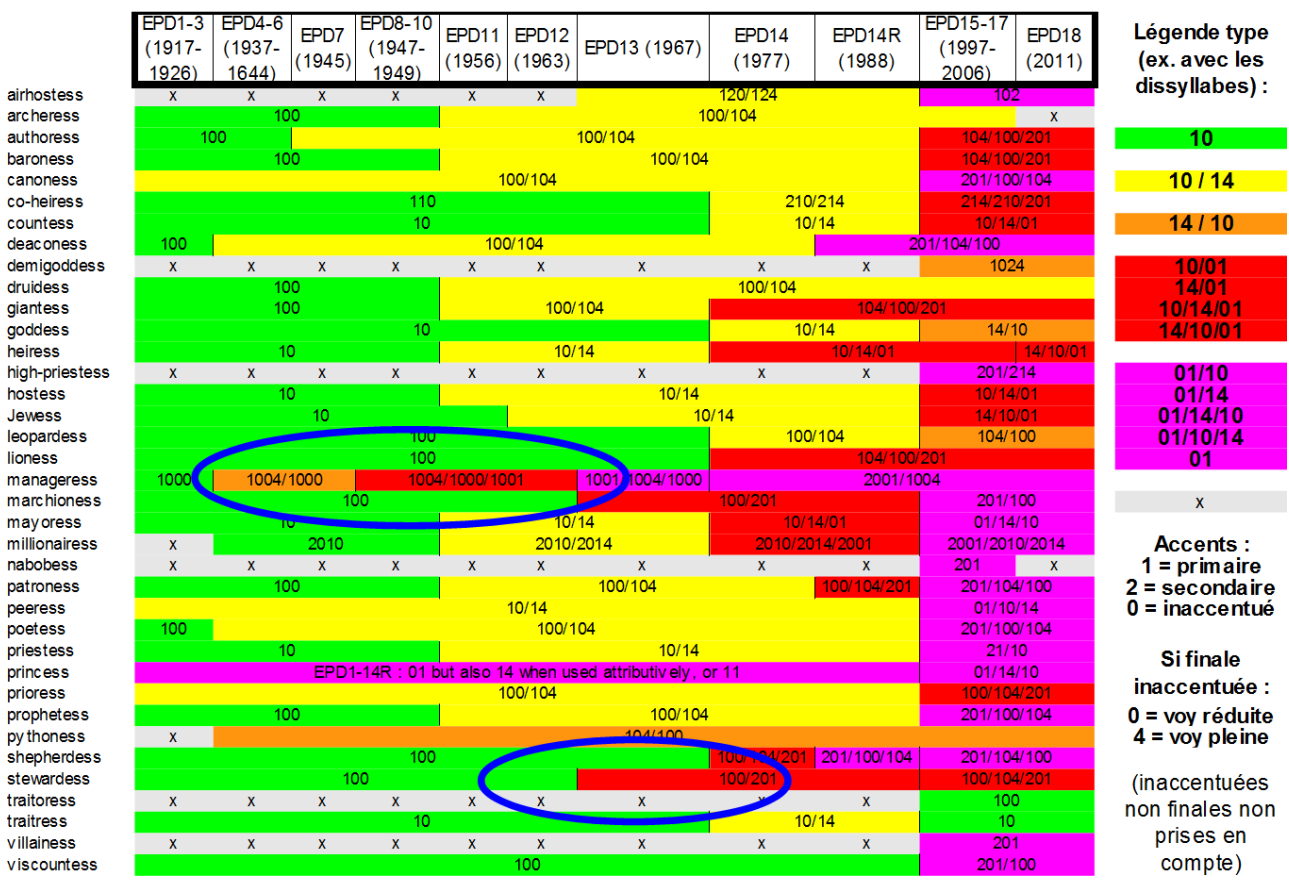


Illustration 4 : Corpus de travail au XXe siècle (dérivés imparisyllabiques uniquement). Les zones entourées correspondent respectivement à manageress et à stewardess.

Manageress et stewardess, deux cas de sur-spécification agentive (manager est normalement neutre, steward est plutôt masculin), sont des dérivations sémantiquement très marquées, symboles de la féminisation des métiers, y compris à un haut niveau de responsabilité impliquant de diriger des hommes (manageress). Il n'est habituellement pas nécessaire de préciser le sexe du manager (la fonction prime sur le sexe), et l'on utilise habituellement plutôt hostess que stewardess, en particulier dans le cas des hôtesses de l'air. On peut ainsi voir un lien entre le fait que manageress ait été affecté par l'accentuation finale au tout début du XXe siècle et différents éléments historiques, à savoir :

74 - la première vague féministe ; cf. notamment le mouvement des Suffragettes (lutte pour l'égalité des droits) à la fin du XIXe siècle et au début du XXe siècle ;

75 - le première guerre mondiale, où les femmes ont dû remplacer les hommes dans la vie quotidienne (car ceux-ci étaient partis au front) et donc assumer des tâches jusque là considérées comme masculines (labour des champs, travail dans les usines, réparations, etc.), mais aussi où elles ont joué un rôle de premier plan dans la manufacture et la fourniture aux hommes des armes, munitions, vêtements, etc. nécessaires à la vie au front ;

76 - la seconde guerre mondiale, pour des raisons similaires;

77 - la seconde vague féministe (rejet de l'objectif d'égalité dans le système), dans les années 1960-70.

D'autre part, l'évolution fréquentielle diachronique des mots concernés semble conforter nos hypothèses. Ainsi, Google Ngram Viewer montre que des mots tels que laundress et instructress, qui ne prennent pas l'accent sur la finale, ont bien connu un pic fréquentiel mais celui-ci est antérieur au XXe siècle :

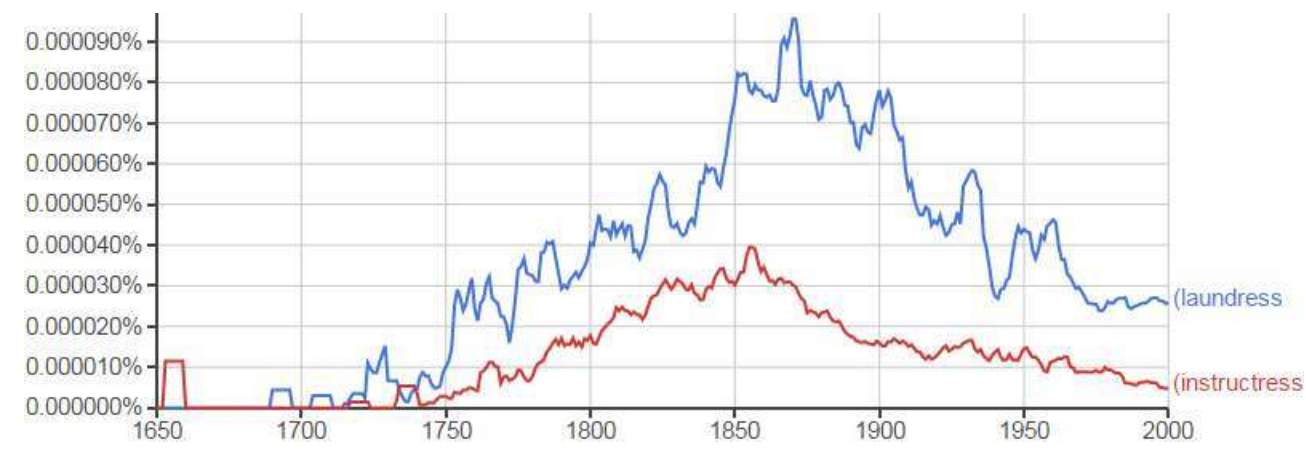


Illustration 5 : Fréquence dans Google Ngram Viewer de laundress et instructress (pluriels inclus) en anglais britannique entre 1650 et 2000

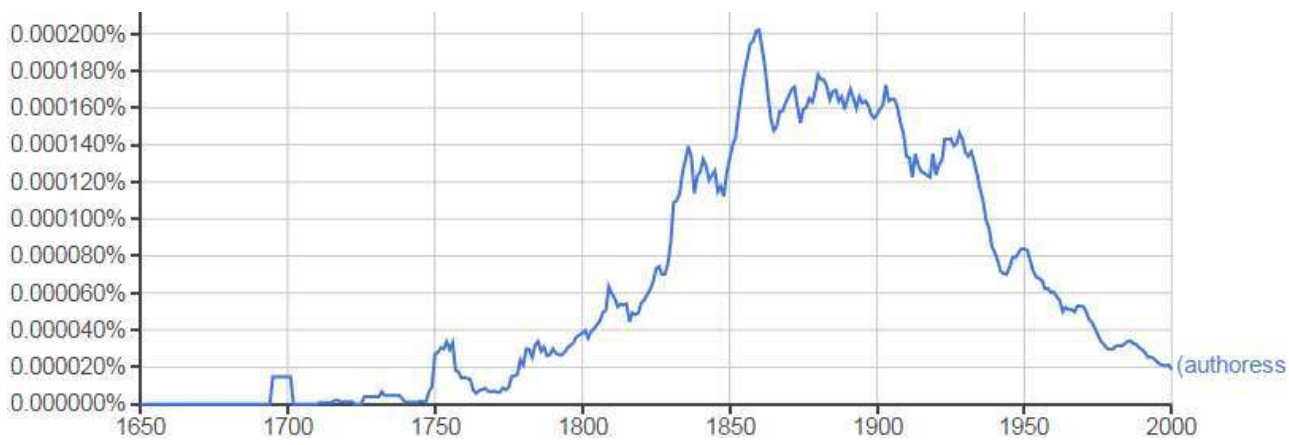

Un nom tel que authoress, qui peut bien prendre l'accent final en variante aujourd'hui, présente également un pic fréquentiel mais celui-ci est observable au milieu du XIXe siècle. Il ne coïncide donc pas avec l'apparition de l'accentuation finale pour ce mot à partir de EPD15 (1997) seulement :

Illustration 6 : Fréquence dans Google Ngram Viewer de authoress (pluriel inclus) en anglais britannique entre 1650 et 2000

En revanche, l'augmentation fréquentielle de l'emploi de manageress semble bien compatible avec l'apparition de la voyelle pleine dans EPD4 (1937) et de l'accent final dans EPD8 (1947) :

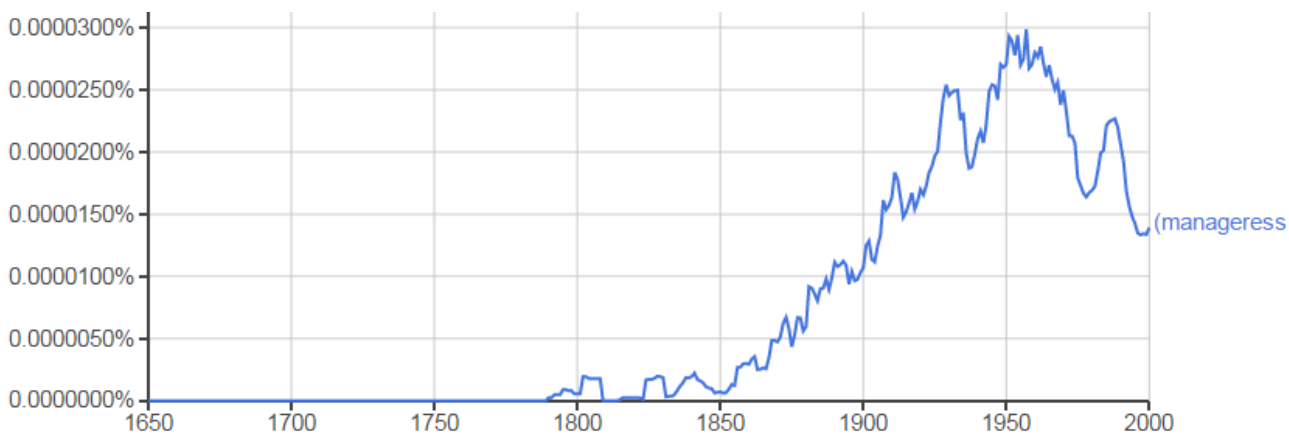

Illustration 7 : Fréquence dans Google Ngram Viewer de manageress (pluriel inclus) en anglais britannique entre 1650 et 2000

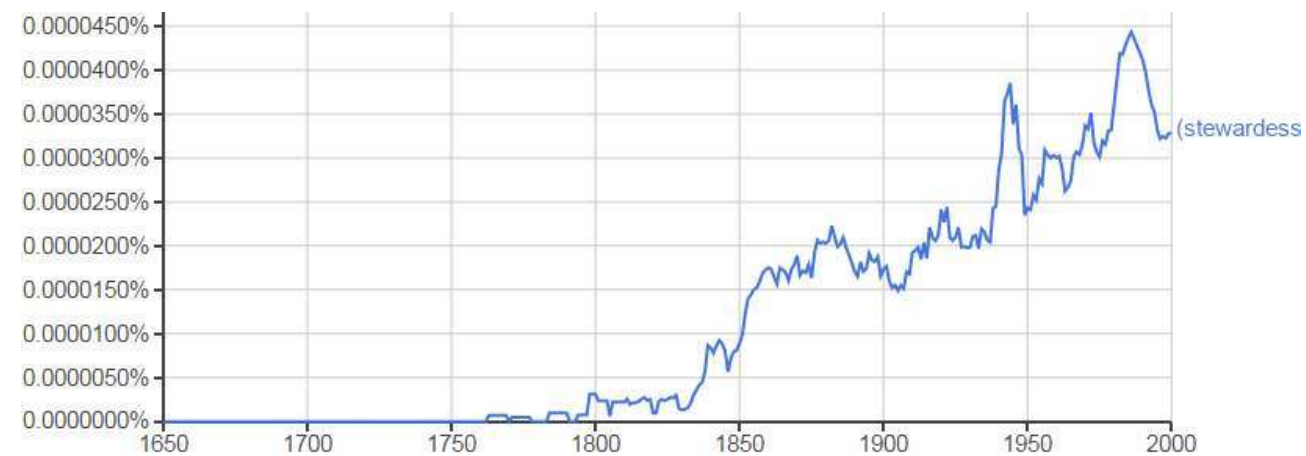

Illustration 8 : Fréquence dans Google Ngram Viewer de stewardess (pluriel inclus) en anglais britannique entre 1650 et 2000

Il en va de même pour stewardess, qui adopte l'accent final dans EPD13 (1967) : 
81 Dans ce dernier cas, il est d'ailleurs fort probable que l'apparition du terme pour renvoyer spécifiquement à une hôtesse de l'air explique la plus forte augmentation fréquentielle du terme à la fin de la première moitié du siècle, après un plateau depuis 1850. L'OED fournit ainsi la date de première attestation des différentes acceptions de stewardess :

a. (1631) A female who performs the duties of a steward; also fig.

b. (1837) A female attendant on a ship whose duty is to wait on the women passengers.

c. (1931) A female attendant on a passenger aircraft who attends to the needs and comfort of the passengers; = air hostess $\mathrm{n}$. at air n.1 Compounds 2. Also, a similar attendant on other kinds of passenger transport.

Dans l'ensemble, il nous semble donc que la féminisation des métiers, mais aussi la recrudescence de la conscience féministe (qu'elle se fasse avec adhésion ou rejet) a pu conduire à l'usage des noms féminins comme un outil de revendication, ou au contraire comme un procédé de stigmatisation dans la langue. Trevian, qui soulevait l'idée d'un effet contrastif de -ess (cf. supra), sous-entend d'ailleurs la même chose en affirmant : « Il est vrai que les luttes féministes ont beaucoup contribué à entacher ces suffixés d'une connotation sexiste » $(2010: 247)$. Constatant le statut précurseur de ces noms précis au sein de leur classe, on peut donc supposer que le phénomène d'accentuation finale des noms féminins en -ess a pu être initié par des considérations féministes ou antiféministes, en accord avec le contexte historique et social de l'époque. Ce n'est que plus tard qu'il a pu se diffuser en affectant d'autres types de mots sur des critères avant tout morphologiques, sans stigmatisation obligatoire (shepherdess, heiress, etc.).

\section{Conclusion}

83 À travers cette étude de l'ensemble de 78 noms féminins en -ess, nous avons montré que le critère morphologique est primordial dans leur accentuation, puisque si les dérivés parisyllabiques ont peu de chances de recevoir un accent final (type governess), ceux où ess est un ajout transparent sont au contraire fortement sujets à un schéma oxyton (type poetess).

84 Nous avons également montré que les cas de dérivation transparente par ajout d'une syllabe correspondent systématiquement à des cas de sur-spécification agentive, renforçant le potentiel accentuable du suffixe. À l'inverse, les dérivés parisyllabiques évitent ou gomment cette sur-spécification, ce qui les rend ainsi sémantiquement peu aptes à être accentués sur la finale. Pourtant, aux interstices de la morphologie, la sémantique peut prendre le pas et conduire malgré tout à une accentuation finale lorsque la propriété «féminin » peut paraître notionnellement surprenante ou inhabituelle, opposant ainsi le type governess (genre social féminin, pas d'accent final) à murderess (genre social masculin, accent final possible). Même en l'absence d'accent final, il est probable que la distinction entre voyelle finale pleine ou réduite joue un rôle similaire (abbess vs seamstress).

85 Tous ces mots conservent néanmoins probablement un potentiel contrastif, sur le plan extra-linguistique mais aussi purement linguistique. On peut ainsi s'attendre à un accent final sur mayoress dans un énoncé tel que :

Apparently the Mayor last year or the Mayoress er was rather keen and organized a match between the Dulwich Club and her team nominally which turned out to be made up of masters from Dulwich College. (BNC) 

renvoyer soit à une femme occupant la fonction désignée (accent final attendu), soit à la femme de l'homme occupant cette fonction (accent final improbable). Inversement, les cas pour lesquels il est habituel, naturel ou socialement accepté de distinguer le masculin $\mathrm{du}$ féminin (mistress, waitress, shepherdess, leopardess, etc.) sont peu enclins à prendre l'accent sur la finale. Nous avons ainsi pu établir une gradation sémantique selon la situation sociale des signifiés, qui permet de prédire le placement de l'accent en conjonction avec la morphologie des signifiants.

diffusion lexicale, mais que, contrairement à la caractérisation du phénomène par Yang
(1969), il était ici phonétiquement graduel. Par ailleurs, nous avons expliqué que ce changement accentuel trouvait possiblement sa source dans le contexte socio-historique du début du XXe siècle, la terminaison -ess pouvant représenter un outil de revendication féministe (dont témoigne la création même du suffixé ,Suffra'gette) ou de stigmatisation. Cela est appuyé par le statut précurseur des noms manageress et stewardess, avant la diffusion subséquente du changement au reste de la classe en suivant des critères avant tout morphologiques.

S'agissant d'un changement en cours (qui concerne l'anglais britannique mais pas l'anglais américain), seul l'avenir dira si le changement s'opérera jusqu'au bout dans le cas des dérivés imparisyllabiques, avec une possible disparition de l'accentuation du radical. Cela semble néanmoins en bonne voie, puisque EPD18 et/ou ODP recensent à présent plusieurs items à accentuation finale exclusive (villainess, manageress, marchioness, etc.). Trevian conclue la même chose:

Judging from the data delivered by LPD1/2 and EPD15, conversion of all -ess items to final stressing may be under way today, at least in British English. (2007:446)

Il nous semble toutefois moins sûr que le phénomène atteigne massivement les dérivés parisyllabiques, à moins que ceux-ci ne finissent par se plier au modèle que pourrait alors représenter la sous-classe des dérivés imparisyllabiques.

Quoi qu'il en soit, nous avons montré que, au-delà des simples considérations morphologiques, l'avancée de l'accent sur la terminaison répondait à des considérations sémantiques. C'est pour cette raison que nous réfutons «l'influence de la terminaison contraignante -C'C'e » (type finesse) supposée par J.-M. Fournier (2010 : 38 ; cf. supra). Par ailleurs, il indiquait explicitement en 2002 que les noms féminins en -ess devaient s'appréhender au sein du même ensemble lexical que ca'ress :

Si la référence à [la terminaison $\left\langle(\mathrm{V}) \mathrm{C}^{\prime} \mathrm{C}^{\prime} \mathrm{e} \#>\right.$ ] est bien la clé de l'accentuation exceptionnelle de [caress, duchess(e), duress(e), prowess, cuirass, harass, morass, abyss et possess], la question qui se pose alors, c'est de comprendre pourquoi <ss\#> lui est assimilée. Formulée ainsi, la réponse apparaît en fait relativement évidente : en effet, en particulier en raison de l'évolution du suffixe féminin, une large majorité des mots en <sse\#> a perdu son <e\#>; réciproquement, la quasi-totalité des mots en $<s$ s\#> provient de <ss\#>, en excluant bien entendu les bases monosyllabiques, quelle que soit la structure dans laquelle elles figurent, et les deux suffixes germaniques less et -ness. C'est ce que confirment d'ailleurs les deux cas de morass et abyss, d'abord écrits morasse et abysse. L'ensemble de ces éléments contribue donc ainsi à analyser l'existence d'une assimilation de <ss\#> à <sse\#>, assimilation confirmée par la variation graphique synchronique $<(e) \#>$ attestée pour duchess $(e)$, duress $(e)$ et largess $(e)$.

[...] En faisant ainsi des mots en <ess\#> la source du processus de discrimination, on est donc conduit à considérer que c'est par extension, fondée sur les 
caractéristiques distinctives de $\langle\mathrm{s}\rangle$, qu'il en est venu à affecter l'ensemble des unités en <Vss\#>. (2013:69-70)

91 En réalité, la plupart des dissyllabes considérés par Fournier comme exceptionnels nous semblent avoir une accentuation justifiable par ailleurs : morass n'a pas été emprunté directement au français ${ }^{14}$, abyss et possess sont des préfixés latins ${ }^{15}$, et duchess et duchesse sont deux mots distincts ${ }^{16}$. Quant à caress, sa période d'emprunt (première attestation en 1651 pour le nom et 1679 pour le verbe selon l'OED) peut justifier le maintien de l'accentuation finale française ${ }^{17}$ tandis que les autres mots en <-ss\#> de la liste (sauf harass) datent des XIVe, XVe ou XVIe siècles. Le cas de cuirass, qui remonte à 1464, est particulier car son accentuation finale est due à un réemprunt ultérieur au français tandis qu'il n'était pas accentué sur la finale au départ ${ }^{18}$. Enfin, l'accentuation originelle de duress (1320, mais qui s'est également toujours orthographié duresse), prowess (1300) et harass (1618) est [10], leur schéma [01] n'étant également qu'un développement récent ${ }^{19}$. Par opposition, les mots dont J.-M. Fournier (ibidem) s'étonne qu'ils ne prennent pas l'accent final (fortress, buttress, mattress, harness, cypress, carcass, cutlass, canvass, bugloss) n'ont en fait pas de raison de le faire car il s'agit d'emprunts remontant au XIVe siècle (XVIe siècle pour cutlass et bugloss). Ce sont donc seulement duress, prowess et harass qui font partiellement figure d'exceptions isolées, s'appuyant probablement sur le modèle représenté par le type ca'ress/cui'rass. Par conséquent, l'inclusion des noms féminins en ess dans la classe plus large des mots en <ss\#> nous paraît injustifiée.

\section{BIBLIOGRAPHY}

CASTANIER, J. (2010). Vers un nouvel équilibre rythmique en anglais contemporain. L'évolution du placement accentuel dans les mots terminés par de longues séquences post-toniques. Mémoire de Master 2 sous la direction de M. Jean-Louis Duchet. Université de Poitiers.

CASTANIER, J. (2016). L'évolution accentuelle du lexique anglais contemporain appréhendée à travers les dictionnaires de prononciation (XVIIIe - XXIe siècles). Thèse de doctorat sous la direction de M. Jean-Louis Duchet et Mme Sylvie Hanote. Université de Poitiers.

DAHAK, A. (2011). Étude diachronique, phonologique et morphologique des syllabes inaccentuées en anglais contemporain. Thèse de doctorat sous la direction de M. Alain Deschamps. Université Paris 7.

DANIELSSON, B. (1948). Studies on the Accentuation of Polysyllabic Latin, Greek, and Romance LoanWords in English with special reference to those ending in -able, -ate, -ator, -ible, -ic, -ical and ize. Stockholm : Almqvist \& Wiksells.

DUCHET, J.-L. (1994a). Code de l'anglais oral. 2e éd. Gap : Ophrys.

FOURNIER, J.-M. (2010). Manuel d'anglais oral. Gap : Ophrys.

FOURNIER, J.-M. (2013). « Why ca'ress ? » in 11e colloque d'avril sur l'anglais oral - La prosodie. Actes du colloque de Villetaneuse (ALOES) de 2002.61-72. Paris : APLV / Les langues modernes. FoWLER, H. W. (1926). A Dictionary of Modern English Usage. Oxford: Clarendon Press. 
GOWERS, E. (1965). Fowler's Modern English Usage. 2nd edition. Oxford: Oxford University Press. GINÉsY, M. (2004). Mémento de phonétique anglaise. Paris : Armand Colin.

GREENE, E. (1972). “Emily Dickinson Was a Poetess”, in College English, vol. 34, No. 1, 63-70.

HeLlinger, M. (2006). "Sexist Language”, in Encyclopedia of Language and Linguistics. 2nd ed. vol. XI, 265-272. Oxford: Elsevier.

HolmES, J. (1993). "Sex-Marking Suffixes in Written New Zealand English" in American Speech, Vol 68, No. 4, 357-370.

KASTOVSKY, D. \& DALTON-PUfFER, C. (2002). "Sexist German- non-sexist English or non-sexist German-sexist English? Historical observations on a pragmatic question" in Language Sciences 24, 285-296.

TOURNIER, J. (2004). Précis de lexicologie anglaise. Paris : Ellipses.

TRevian, I. (2003). Morphoaccentologie et processus d'affixation de l'anglais. Berne: Peter Lang.

TREVIAN, I. (2007), "Stress-Neutral Endings in Contemporary British English: an Updated Overview", in Language Sciences 29, 426-450.

TREVIAN, I. (2010). Les affixes anglais, productivité, formation de néologismes et contraintes combinatoires. De la diachronie à la synchronie. Berne : Peter Lang.

YANG, W. S.-Y. (1969). « Competing Changes as a Cause of Residue » in Language, vol. 45, No 1, 9-25.

ZUMSTEIN, F. (2007). Variation accentuelle, variation phonétique : étude systématique fondée sur des corpus lexico-phonétiques informatisés anglais. Thèse de doctorat sous la direction de $\mathrm{M}$. Jean-Louis Duchet. Université de Poitiers.

Google Ngram Viewer : https://books.google.com/ngrams (dernière consultation : 25 déc. 2015)

Oxford English Dictionary en ligne : http://www.oed.com/ (dernière consultation : 25 déc. 2015) Sources primaires (corpus)

[BAiley] BAILey, N. (1727). An Orthographical Dictionary, Shewing both the Orthography and the Orthoepia of the English Tongue. London: for T. Cox.

[BARCLAY] BARCLAY, J. (1824). A Complete and Universal English Dictionary. 4e édition (1e publication 1774). London: for William Baynes and Son.

[BOYER 1829] BOYER, A. et al. (1829). Dictionnaire anglais-français et français-anglais. Nouvelle édition (1e publication 1702). Paris : chez Ledentu.

[BOYER 1837] BOYER, A. et al. (1837). Dictionnaire anglais-français et français-anglais. Abrégé de Boyer. Paris: Charles Hingrey.

[BuCHANAN] BuchanAN, J. (1766). An Essay towards Establishing a Standard for an Elegant and Uniform Pronunciation of the English Language. London: for Edward and Charles Dilly.

[CHAMBERs] DonALD, J. (1872). Chambers's Etymological Dictionary of the English Language. 2e édition (1e publication 1867). London, Edinburgh: Chambers.

[CPDBAE] LEWIS, J. W. (1972). A Concise Pronouncing Dictionary of British and American English. Oxford: OUP.

[Dyche] Dyche, T. (1759). A New General English Dictionary. 10e édition (1e publication 1735). London: for C. Ware. 
[ENTICK] ENTICK, J. (1798). New Spelling Dictionary Teaching to Write and Pronounce the English Tongue. 1e édition 1764. London: for C. Dilly.

[EPD1-12] JoNES, D. (1917-1963). English Pronouncing Dictionary. London : Dent \& Sons. 12 éditions.

[EPD13-14] JonES, D \& GIMSON, A. C. $(1967$; 1977). English Pronouncing Dictionary. London: Dent \& Sons. Éditions 13-14.

[EPD14R] JonEs, D., GIMSON, A. C. \& S. RAMSARAN (1988). English Pronouncing Dictionary. London : Dent \& Sons. Réimpression de la 14e édition révisée avec supplément.

[EPD15-18] ROACH, P. et al. (1917-2011). Cambridge English Pronouncing Dictionary. Cambridge: Cambridge University Press. Éditions 15 à 18.

[Fulton \& KNiGHT] Fulton, G. \& KNiGht, G. (1833). A General and Explanatory Dictionary of the English Language. 1E publication 1802. London: Whittaker, Treacher \& Arnot.

[FENNING] FenNing, D. (1771). The Royal English Dictionary, or a Treasury of the English Language. 4e édition (1e publication 1761). London: for L. Hawes et al.

[JAMESON] JAMESON, R. S. (1827). A Dictionary of the English Language. London: William Pickering. [Johnson] Johnson, S. (1756). A Dictionary of the English Language. London: for J. Knapton et al.

[LPD1-3] WELLS, J. C. (1990-2008). Longman Pronunciation Dictionary. Harlow: Longman. 3 éditions.

[ODP] Upton, C., KRETZSChMAR W. A. Jr, KonopkA, R. (2001). Oxford Dictionary of Pronunciation for Current English. New York: OUP.

[PERRY] PerRY, W. (1800). The Royal Standard English Dictionary. 5e édition (1e publication 1775). Boston: Isaiah Thomas and Ebenezer T. Andrews.

[SHERIDAN] SHERIDAN, T. (1780; 1797). A Complete Dictionary of the English Language, Both with Regard to Sound and Meaning. 1e et 4e éditions. London: for C. Dilly.

[S. JONES] JONES, S. (1804). A General Pronouncing and Explanatory of the English Language. 9e édition (1e publication 1798). London: for Vernor and Mood.

[SPIERS 1877] SPIERS, A. (1877). Dictionnaire général anglais - français. 26e édition (1e publication 1846). Paris : Librairie européenne de Baudry.

[SPIERS \& SURENNE 1886] SPIERs, A. \& SURENNE, G. (1886). Standard Pronouncing Dictionary of the French and English Languages in two parts. School edition. 1e publication 1846 (ou 1840). NewYork: D. Appleton and Company.

[SPIERS \& SURENNE 1891] SPIERS, A. \& Quackenbos, G. P. (1891). Spiers and Surenne's French and English Pronouncing Dictionary. 1e publication 1852. New-York: D. Appleton and Company.

[WALKER] WALKeR, J. (1842). A Critical Pronouncing Dictionary and Expositor of the English Language. 1e publication 1791. London: Robinson, Cadell \& Davies.

[WORCESTER 1860] WORCESTER, J. E. (1860). Dictionary of the English Language. Boston: Hickling, Swan and Brewer.

[WORCESTER 1874] WORCESTER, J. E. (1874). A Comprehensive Dictionary of the English Language. Boston: Brewer \& Tileston. 
[WRIGHT] WRIGHT, T. (1852-56). The Universal Pronouncing Dictionary and the General Expositor of the English Language. London : London Printing and Publishing Company.

\section{NOTES}

1. Je tiens à remercier Jean-Louis Duchet et Bernard Magnouloux pour leur relecture attentive de cet article et pour leurs remarques et suggestions.

2. Dont 7 qui, présents dans EPD jusqu'à la 14e édition, ont cependant disparu dans les éditions suivantes, et sont également absents de LPD. Il s'agit de ancress, arbitress, avengeress, dauphiness, foundress, idolatress et inspectress.

3. Alors qu'une voyelle entravée en syllabe post-tonique est idéalement réduite, comme dans mattress, cypress, etc. ou dans les suffixes -less et -ness.

4. Bien qu'il s'agisse d'un dictionnaire américain, les transcriptions fournies par Worcester (1860), ainsi que par d'autres lexicographes du XIXe siècle tels que Noah Webster, ne correspondent en réalité pas vraiment, à l'époque, à des prononciations américaines. Nous détaillons en particulier cette observation dans Castanier (2016: 99-107).

5. EPD14R : 14e édition révisée avec supplément, publiée en 1998 par Susan Ramsaran.

6. Seuls $35,2 \%$ des mots peuvent prendre l'accent final dans ODP, mais il nous apparait de façon générale que ce dictionnaire est moins sensible à la variation que EPD et LPD. Quant à CPDBAE, qui fournit un peu plus de schémas [-1] que EPD14(R), il permet de supposer que EPD était un peu en retard dans son enregistrement lexicographique du phénomène.

7. Seducer et seductress ne sont pas en lien de dérivation direct, puisque la forme féminine devrait dériver, à strictement parler, de * seductor.

8. Nous plaçons druidess et leopardess entre parenthèses car ils peuvent bien recevoir un accent final selon ODP et/ou CPDBAE, mais pas selon EPD et LPD.

9. Désignant vraisemblablement une personne refoulée d'un établissement par un videur.

10. On peut cependant suspecter qu'il puisse y avoir une distinction entre lioness désignant un lion femelle, et lioness désignant une femme dont le comportement est comparé à celui d'une lionne.

11. La double accentuation primaire était d'ailleurs un procédé fréquemment employé dans certains dictionnaires antérieurs au XXe siècle lorsque leurs auteurs ne pouvaient trancher en faveur d'une accentuation ou d'une autre. Il s'agissait souvent de mots composés, mais aussi de mots en cours de changement accentuel.

12. L'accentuation des adverbes en -arily fera également l'objet d'un article à paraitre (sous réserve d'un changement de titre de l'ouvrage) : "Not necessarily neutral : placement accentuel dans les dérivés en -orily et -arily» in HANOTE, S. \& NITA, R. (eds) Morphophonologie, lexicologie et langue de spécialité. Presses Universitaires de Rennes.

13. Par exemple, dans ses développements les plus récents, necessarily ne signifie plus seulement « in a necessary manner " (surtout dans la locution négative « not necessarily »), contrairement à un adverbe du type hereditarily ( in a hereditary manner »).

14. "Either < Middle Low German mōras or < Middle Dutch morasch (also in forms maras, marasch ; Dutch moeras (1599 in Kiliaan)), both ultimately < Old French marais , mares , maresc , etc." (OED). Ceci ne suffit pas nécessairement à justifier l'accent final de ce mot, mais cela empêche en tout cas par principe de l'inclure d'office dans les emprunts au français, et donc de l'inclure dans le raisonnement.

15. Possess n'est en réalité pas préfixé mais, comme le souligne Fournier (2013: 64), sa seconde syllabe s'interprète aisément comme une pseudo-base aux côtés de obsess et assess. 
16. L'argument de J.-M. Fournier est douteux pour duchess(e) puisqu'il ne s'agit pas de variation graphique, duchess et duchesse n'ayant nullement le même sens. De plus, selon l'OED, duchesse (1794) est largement postérieur à duchess (1395).

17. Danielsson (1948: 29) considère la date approximative de 1660 comme un pivot avant lequel les emprunts au français ont été dans l'ensemble intégrés au lexique anglais, et après lequel ils ont conservé leur accentuation finale (indépendamment du possible phénomène plus récent de rétraction accentuelle). Nos travaux dans Castanier (2016, chapitre 3 en particulier) confirment parfaitement cette observation.

18. "In 16th cent. a frequent English form was curats, cuirats, apparently under the influence of Italian curazza: compare Middle Low German koritz, Old Danish körritz, kyrritz, etc. This being, from its final $s$, treated as a plural, gave the mutilated singular curat, curate, etc., common 15601650. The stress was then on the first syllable, but was subsequently under French influence shifted to the second [...]" (OED)

19. Notre corpus de travail dans Castanier (2016) montre que duress n'était accentué que [10] au XVIIIe siècle, et si [01] est apparu marginalement au XIXe siècle, ce schéma n'est devenu majoritaire qu'au XXe siècle. Quant à prowess et harass, leur variante [01] n'apparaît qu'à la fin du XXe siècle avec la publication de EPD15 (1997). L'accentuation finale de harass en anglais britannique semble être un américanisme, puisque l'accent final semblait déjà exister en anglais américain dès le début du XXe siècle. Il n'existe en revanche pas pour prowess dans cette variété.

\section{ABSTRACTS}

Within a dynamic synchronic approach using in particular all 18 editions of the English Pronouncing Dictionary, this study examines the emergence since the mid-20 th century of final stress in British English (but not in American English) on feminine nouns in -ess (authoress, shepherdess, actress, manageress, etc.), whereas this ending used to be perfectly stress-neutral. The situation seems confusing, since $41 \%$ of the 78 contemporary feminine nouns in -ess now accept final stress as their main or variant pattern. When unstressed, the final syllable also oscillates between a full vowel [-es] and a weak vowel [-Is/-əs].

We show that morphology plays a fundamental role in this new pattern, depending whether the masculine or neutral deriving forms and the feminine derivatives are parisyllabic (stress is usually not shifted: 'actor > 'actress) or imparisyllabic (final stress is frequent: 'author > ,autho'ress). We also show that this ending may have a marked meaning, and that this semantic criterion interacts with the morphological criterion in attracting stress on the final syllable or not. In the final part we try to determine the potential origins of the change by identifying its lexical diffusion pattern, which suggests the likely role of the socio-historical context (wars, feminist waves, etc.).

Dans une approche de synchronie dynamique utilisant notamment les 18 éditions de l'English Pronouncing Dictionary, cette étude examine l'apparition, depuis le milieu du XXe siècle, d'un accent final en anglais britannique (mais non en anglais américain) sur les noms féminins en -ess (authoress, shepherdess, actress, manageress, etc.), terminaison autrefois parfaitement neutre accentuellement. La situation semble confuse, puisque $41 \%$ des 78 noms féminins en -ess contemporains admettent aujourd'hui un accent final en schéma principal ou en variante. Lorsqu'elle n'est pas accentuée, la finale oscille par ailleurs entre une voyelle pleine [-es] ou 
réduite [-Is/-əs].

Nous montrons que la morphologie régule fortement cette nouvelle accentuation, selon que dérivants masculins ou neutres et dérivés féminins sont parisyllabiques (l'accent n'est alors généralement pas final : 'actor > 'actress) ou imparisyllabiques (l'accent est alors souvent final : 'author > autho'ress). Par ailleurs, nous montrons que le sémantisme de la terminaison féminine ess est parfois marqué, et que ce critère sémantique interagit avec le critère morphologique dans l'attraction ou non de l'accent sur la finale. Enfin, nous proposons de remonter aux sources possibles du changement en identifiant son schéma de diffusion lexicale, qui pointe le rôle probable du contexte socio-historique (guerres, vagues féministes, etc.).

INDEX

Keywords: English phonology, stress placement, feminine nouns, -ess ending, ongoing change, morphology, semantics, lexical diffusion

Mots-clés: phonologie de l'anglais, accentuation, noms féminins, terminaison -ess, changement en cours, morphologie, sémantique, diffusion lexicale

\section{AUTHOR}

\section{JÉRÉMY CASTANIER}

Laboratoire CORPUS (EA 4295), Université de Picardie Jules Verne 